Supporting Information for

\title{
Extreme in-plane thermal conductivity anisotropy in titanium trisulfide caused by heat-carrying optical phonons
}

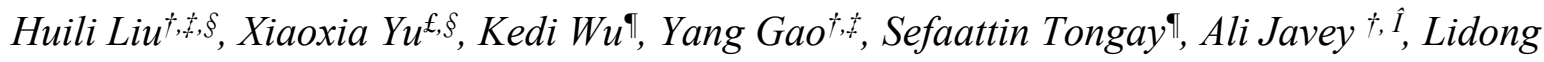

Chen ${ }^{\epsilon, *}$, Jiawang Hong ${ }^{£, *}$ and Junqiao Wü,t,**

${ }^{\dagger}$ Materials Sciences Division, Lawrence Berkeley National Laboratory, Berkeley, California 94720, USA

Department of Materials Science and Engineering, University of California, Berkeley, California 94720, USA

${ }^{£}$ School of Aerospace Engineering, Beijing Institute of Technology, Beijing 100081, China

\School for Engineering of Matter, Transport, and Energy, Arizona State University, Tempe, Arizona 85287, USA

ÎDepartment of Electrical Engineering and Computer Science, University of California, Berkeley, California 94720, USA

${ }^{€}$ State Key Laboratory of High Performance Ceramics and Superfine Microstructure, Shanghai Institute of Ceramics, Chinese Academy of Sciences, Shanghai, 200050, China

$\S$ Those authors contributed equally to this work

*Author to whom correspondence should be addressed. Electronical mail: wuj@berkeley.edu, hongjw@bit.edu.cn, cld@mail.sic.ac.cn 
Experimental details. $\mathrm{Ti}_{3}$ nanoribbon device fabrication. $\mathrm{TiS}_{3}$ single crystals, shown in Figure $\mathrm{S} 1$, grow naturally into micro-ribbons or whiskers parallel to its b-axis direction. ${ }^{1}$ As reported previously, ${ }^{2,3} \mathrm{TiS}_{3}$ flakes were mechanically exfoliated onto a $\mathrm{SiO}_{2} / \mathrm{Si}$ substrate using PDMS (Polydimethylsiloxane), and then tailored into nanoribbons $(0.5-2 \mu \mathrm{m}$ in width and $25-40 \mu \mathrm{m}$ in length) using electron-beam lithography (EBL) followed by reactive ion etching (RIE). Specifically, the flakes were coated by PMMA (Poly(methyl methacrylate), C4-950, 4000 rpm) and baked at $180^{\circ} \mathrm{C}$ for 5 mins. The PMMA was patterned with EBL followed by a developing process using MIBK : IPA = $1: 3$ for 1 min. The exposed $\mathrm{TiS}_{3}$ was etched via RIE using a mixed gas $\left(90 \% \mathrm{SF}_{6}\right.$ and $\left.10 \% \mathrm{O}_{2}, 60 \mathrm{sccm}\right)$ for several seconds. After the PMMA removal with acetone and rinsing by isopropyl alcohol, $\mathrm{TiS}_{3}$ nanoribbons were obtained. The thickness of the nanoribbon was confirmed by Atomic Force Microscope (AFM), as shown in Figure 2 and Figure S10. Selected individual nanoribbon was manually dry-transferred onto an empty, suspended-pad micro-device using a sharp Tungsten needle (600 nm tip diameter, Cascade Microtech). The electrical quality of the electrodes was verified by a linear I-V relationship (ohmic contact). To further improve the electrical and thermal contact, a small amount of Pt metal was deposited by focused ion beam (FIB, dual-beam FEI Quanta) to bond the end of the nanoribbon to the underneath metal electrodes (Figure $2 \mathrm{~d}$ and $2 \mathrm{e}$ ). Indeed, the electrical contact resistance is reduced after the FIB Pt deposition, as shown in Figure S2. The devices were annealed at $373 \mathrm{~K}$ for one hour in vacuum to further improve electrical and thermal contacts at the electrodes.

Thermal and electrical measurements. The thermal resistance, electrical conductance, and Seebeck coefficient of nanoribbons were measured using the suspended-pad micro-devices technique following previous reports. ${ }^{4,5}$ Two $\mathrm{SiN}_{\mathrm{x}}$ pads with Pt electrodes were suspended by 
long and flexible $\mathrm{SiN}_{\mathrm{x}}$ arms $(\sim 400 \mu \mathrm{m})$. Pt serpentine electrodes on the pads were for microheater and thermometers, and additional four Pt electrodes were for electrical resistance and thermopower measurements. A DC current $(I=0-15 \mu \mathrm{A})$ was applied to the micro-heater for the temperature raising (hot pad) of $\Delta T_{h}$, and the other pad (cold pad) sensed a temperature of $\Delta T_{c}$ through the nanoribbon. An external heater (Lakeshore 335 Temperature Controller) and cryogenic compressor cooler (HC-4A, Sumitomo CRYOGENICS) controlled the global temperature in the chamber, which was under high vacuum $\left(<10^{-6}\right.$ Torr $)$ during the measurements. An AC current with a small amplitude of $\sim 500 \mathrm{nA}$ and frequency of $1.1 \mathrm{kHz}$ $(199 \mathrm{~Hz})$ was applied to probe $\Delta T_{h}\left(\Delta T_{c}\right)$ on the hot (cold) pad using Lock-in amplifiers. The thermal conductance of the nanoribbon $(G)$ is given by $G=\left(Q \times \Delta T_{c}\right) /\left(\Delta T_{h}^{2}-\Delta T_{c}^{2}\right)$, where $Q$ is the heating power, $Q=I^{2} \times\left(R_{h}+R_{\text {arm }}\right)$, and $R_{h}$ and $R_{\text {arm }}$ are the resistance of heating $\mathrm{Pt}$ electrodes and arms, respectively. Electrical resistance was measured by the four-probe method using a Keithley nanovoltmeter (2182A) and precision current source (6220). The dimensions of nanoribbons were determined by Scanning Electron Microscope (SEM) and AFM. Data errors were estimated as $\sim 8 \%$ for thermal conductivity, and $\sim 5 \%$ for electrical conductivity and Seebeck coefficient. The length dependence of thermal conductance was performed for several nanoribbons from the same flake. Figure $2 \mathrm{f}$ shows cross-sectional area divided by thermal conductance $(A / G)$ as a function of the nanoribbon length $(L)$, where a linear relationship is seen and extrapolated to nearly zero $A / G$ at $L=0$, proving negligible contact thermal resistance for these nanoribbons devices. Figure 3a and Figure S11 show the in-plane thermal conductivity of $\mathrm{TiS}_{3}$ of two devices along a- and b-axis, respectively. Figure S12 shows the measured thermal conductance at $300 \mathrm{~K}$ for Device2_b-axis, where the raising temperature of hot pad and cold pad is $\Delta T_{\mathrm{h}}=0-4.6 \mathrm{~K}$ and $\Delta T_{\mathrm{c}}=0-1.4 \mathrm{~K}$, respectively. The measured heat flow through the 
nanoribbon increases linearly with the temperature difference between hot pad and cold pad, $\Delta T_{\mathrm{h}}$ $-\Delta T_{\mathrm{c}}=0-3.2 \mathrm{~K}$, as shown in Figure $\mathrm{S} 12 \mathrm{~b}$, therefore the thermal conductance is $46.4 \mathrm{nW} \mathrm{K}^{-1}$ at $300 \mathrm{~K}$ for Device2_b-axis.

Theoretical calculation. First-principles electronic structure calculations of $\mathrm{TiS}_{3}$ were performed using density functional theory (DFT) within the generalized gradient approximation (GGA).

The Perdue-Burke-Erznerhof (PBE) form was used for the exchange-correlation functional. ${ }^{6}$ The projector-augmented plane wave basis method as implemented in VASP (Vienna ab initio simulation package) ${ }^{7}$ was used for all structural relaxations and supercell calculations for interatomic force constants. A $8 \times 10 \times 4$ Monkhorst-Pack $k$-point grid and a plane wave energy cutoff of $500 \mathrm{eV}$ was used for all structural relaxations. We used the DFT-D2 method as implemented in VASP for describing the weak vdW interaction between $\mathrm{TiS}_{3}$ layers. The convergence threshold for energy and forces was set to $10^{-7} \mathrm{eV}$ and $10^{-4} \mathrm{eV} / \AA$, respectively. In the supercell calculations for obtaining harmonic (second order) interatomic force constants (IFCs) and anharmonic (third order) IFCs, Phonopy ${ }^{8}$ and ShengBTE code ${ }^{9}$ were used by taking into consideration non-analytical term correction and the force constant calculations were done with a same $2 \times 3 \times 2$ supercell and a $4 \times 3 \times 2 q$-point mesh, until convergence threshold above all was satisfied. The simulated lattice constants: $\mathrm{a}=4.97 \AA, \mathrm{b}=3.93 \AA, \mathrm{c}=8.80 \AA$, are in consistency with literature. ${ }^{10,11}$

\section{Note 1. Phonon dispersion and thermal properties}

The simulated phonon dispersion is shown in Figure S4. No imaginary part exists, indicating thermodynamic stability for the cell. In $\mathrm{TiS}_{3}$, there are 3 acoustic modes and 21 optical modes, corresponding to 8 atoms per primitive unit cell. The phonon dispersions with DFT are 
consistent with experimental Raman data ${ }^{1}$ shown in Figure S4. Indeed, in the energy range of $\sim 10-30 \mathrm{meV}$, phonon modes (optical phonons) are more dispersive along the $\Gamma-\mathrm{Z}$ direction (baxis) than along the $\Gamma$ - Y direction (a-axis). These lattice vibrations are mostly associated with sulfur atoms, as shown in the phonon DOS plot in Figure S4, leading to anisotropy in optical phonon DOS as shown in Figure S6. Above $\sim 30 \mathrm{meV}$, it is seen that all optical phonons are less dispersive, have small group velocity, and do not carry heat. The anisotropy of group velocities is tabulated in Table S1. The average group velocity for optical modes is $1.02 \mathrm{~km} \mathrm{~s}^{-1}$ along the baxis, much higher than $0.49 \mathrm{~km} \mathrm{~s}^{-1}$ along a-axis. Thermal conductivity of optical modes at $300 \mathrm{~K}$ along the b-axis is predicated to $3.74 \mathrm{~W} \mathrm{~m}^{-1} \mathrm{~K}^{-1}$, in stark contrast to $0.97 \mathrm{~W} \mathrm{~m}^{-1} \mathrm{~K}^{-1}$ along a-axis. In conclusion, the low-lying optical modes between $\sim 15-30 \mathrm{meV}$ play the essential role in heat conduction, and dominate the extreme in-plane anisotropy in thermal conductivity of $\mathrm{TiS}_{3}$. Figure $\mathrm{S} 7$ shows the percentage of contribution to $\kappa$ from different phonon modes, where it is shown that significant contributions to thermal conductivity are mainly from the optical modes of No. 4 - 13 in the range of $\sim 15-30 \mathrm{meV}$. The Mode 8 shows a prominent optical vibration with large dispersiveness, and the atomic vibrations of this mode is shown in Figure S9b. Figure S13 shows the cumulative thermal conductivity versus phonon mean free path (MFP) along aand b-axis at $300 \mathrm{~K}, 100 \mathrm{~K}$, and $50 \mathrm{~K}$, respectively. At $300 \mathrm{~K}$, the average phonon mean free path is in the range of $2-10 \mathrm{~nm}$, much smaller than the nanoribbon thickness $(\sim 100 \mathrm{~nm})$. However, at $50 \mathrm{~K}$, the average MFP increases to the range of $50-200 \mathrm{~nm}$, comparable to the thickness of nanoribbons. Therefore, at low temperatures boundary scattering becomes dominant in the phonon transport, leading to large difference between the calculated and measured thermal conductivity in Figure 3a.

\section{Note 2. Born effective charge tensor}


The Born effective charge tensor of the atom $I$, is defined as the induced polarization $(P)$ of the solid along the $\mu$ direction by a displacement $(\delta)$ in the $v$ direction of the sublattice of the atom $I$, which is also described by the linear relationship between the force $(F)$ on the atom and the macroscopic electric field $(E),{ }^{12}$

$$
\left(e^{*}\right)_{I, \mu \nu}=\Omega_{0} \frac{\partial P_{\mu}}{\partial \delta_{I, v}}=\frac{\partial F_{I, v}}{\partial E_{\mu}}
$$

Born effective charge tensor can be calculated using density functional perturbation theory (DFPT) or within the finite-difference method (FDM), which can be used to estimate the polarization according to eq S1. The in-plane Born effective charge tensor for Ti and $\mathrm{S}$ in $\mathrm{TiS}_{3}$ is tabulated in Table S3.

\section{REFERENCES}

(1) Wu, K.; Torun, E.; Sahin, H.; Chen, B.; Fan, X.; Pant, A.; Wright, D. P.; Aoki, T.; Peeters, F. M.; Soignard, E.; Tongay, S. Unusual lattice vibration characteristics in whiskers of the pseudoone-dimensional titanium trisulfide $\mathrm{TiS}_{3}$. Nat. Commun. 2016, 7, 12952.

(2) Lee, S.; Yang, F.; Suh, J.; Yang, S.; Lee, Y.; Li, G.; Sung Choe, H.; Suslu, A.; Chen, Y.; Ko, C.; Park, J.; Liu, K.; Li, J.; Hippalgaonkar, K.; Urban, J. J.; Tongay, S.; Wu, J. Anisotropic inplane thermal conductivity of black phosphorus nanoribbons at temperatures higher than $100 \mathrm{~K}$. Nat. Commun. 2015, 6, 8573.

(3) Liu, H.; Choe, H. S.; Chen, Y.; Suh, J.; Ko, C.; Tongay, S.; Wu, J. Variable range hopping electric and thermoelectric transport in anisotropic black phosphorus. Appl. Phys. Lett. 2017, 111, (10), 102101.

(4) Lee, S.; Hippalgaonkar, K.; Yang, F.; Hong, J.; Ko, C.; Suh, J.; Liu, K.; Wang, K.; Urban, 
J. J.; Zhang, X.; Dames, C.; Hartnoll, S. A.; Delaire, O.; Wu, J. Anomalously low electronic thermal conductivity in metallic vanadium dioxide. Science 2017, 355, 371-374.

(5) Shi, L.; Li, D.; Yu, C.; Jang, W.; Kim, D.; Yao, Z.; Kim, P.; Majumdar, A. Measuring thermal and thermoelectric properties of one-dimensional nanostructures using a microfabricated device. J. Heat Transfer 2003, 125, 881-888.

(6) Perdew, J. P.; Burke, K.; Ernzerhof, M. Generalized gradient approximation made simple. Phys. Rev. Lett. 1996, 77, 3865-3868.

(7) Kresse, G.; Furthmüller, J. Efficient iterative schemes for ab initio total-energy calculations using a plane-wave basis set. Phys. Rev. B 1996, 54, 11169-11186.

(8) Togo, A.; Oba, F.; Tanaka, I. First-principles calculations of the ferroelastic transition between rutile-type and $\mathrm{CaCl}_{2}$-type $\mathrm{SiO}_{2}$ at high pressures. Phys. Rev. B 2008, 78, 134106.

(9) Li, W.; Carrete, J.; A. Katcho, N.; Mingo, N. ShengBTE: A solver of the Boltzmann transport equation for phonons. Comput. Phys. Commun. 2014, 185, 1747-1758.

(10) Zhang, J.; Liu, X.; Wen, Y.; Shi, L.; Chen, R.; Liu, H.; Shan, B. Titanium trisulfide monolayer as a potential thermoelectric material: a first-principles-based boltzmann transport study. ACS Appl. Mater. Interfaces 2017, 9, 2509-2515.

(11) Wu, J.; Wang, D.; Liu, H.; Lau, W.-M.; Liu, L.-M. An ab initio study of TiS 3 : a promising electrode material for rechargeable $\mathrm{Li}$ and $\mathrm{Na}$ ion batteries. RSC Advances 2015, 5, 21455-21463. (12) Gonze, X.; Lee, C. Dynamical matrices, Born effective charges, dielectric permittivity tensors, and interatomic force constants from density-functional perturbation theory. Phys. Rev. B 1997, 55, 10355-10368. 

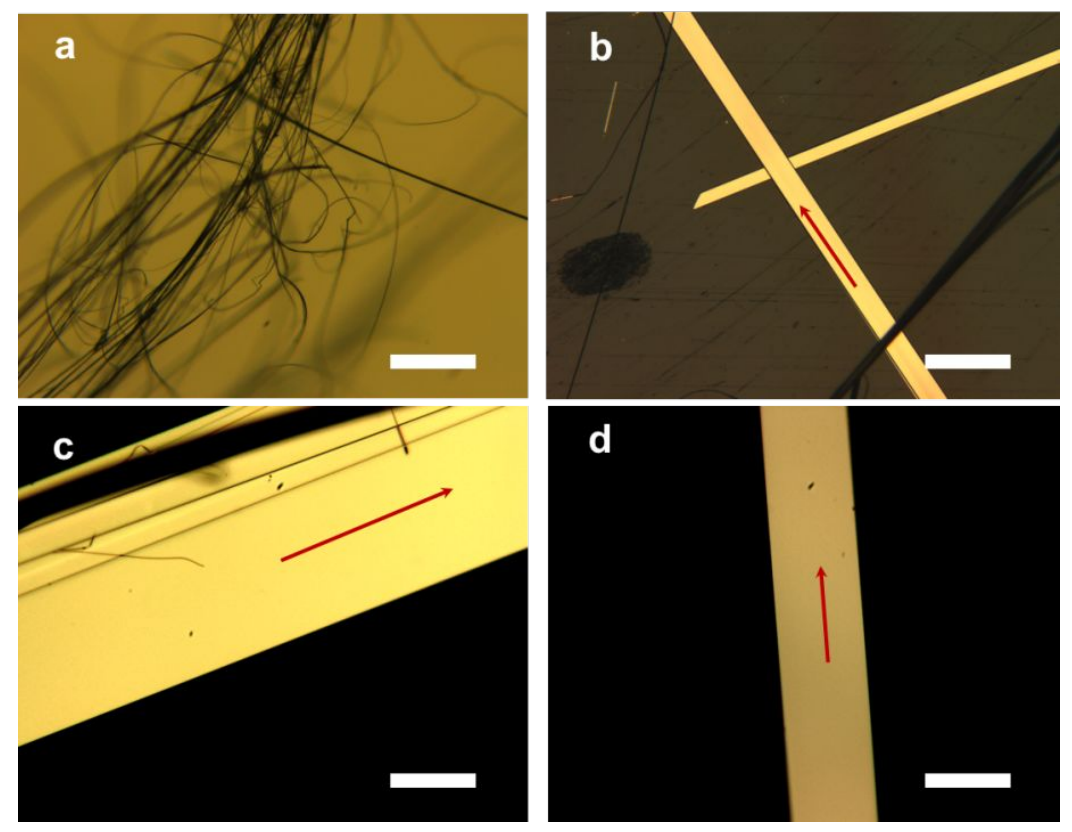

Figure S1. Optic images of $\mathrm{TiS}_{3}$ single crystal whiskers, showing the natural geometric anisotropy. The red arrows indicate the natural chain direction (along the b-axis). Scale bars, 1 $\mathrm{mm}(\mathrm{a}) ; 200 \mu \mathrm{m}$ (b); $50 \mu \mathrm{m}$ (c), (d).

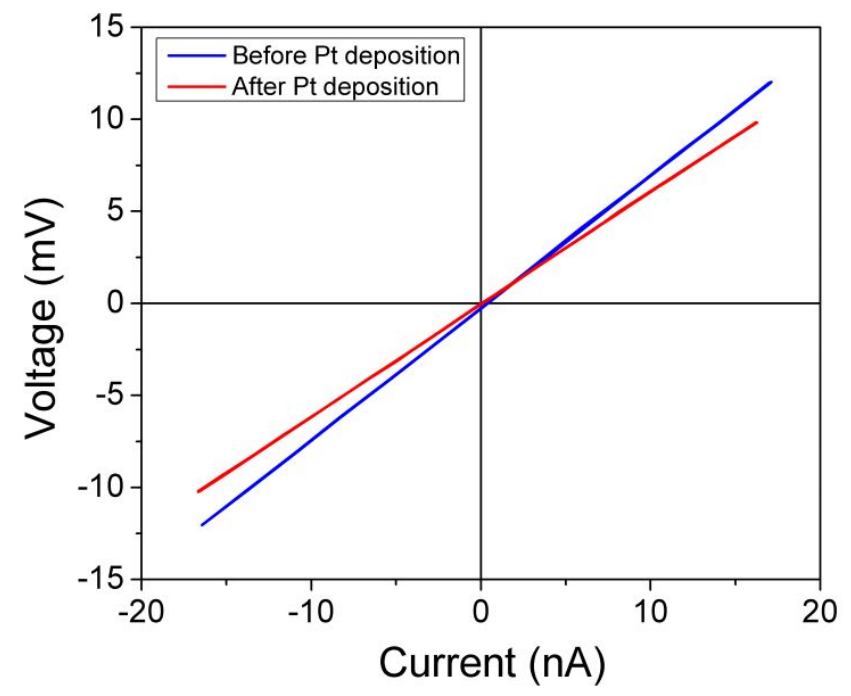

Figure S2. Electrical characterization before and after deposition of a small amount of Pt, bonding the nanoribbon onto the underlying electrodes. The electrical contact resistance is 
reduced after the Pt deposition by FIB.
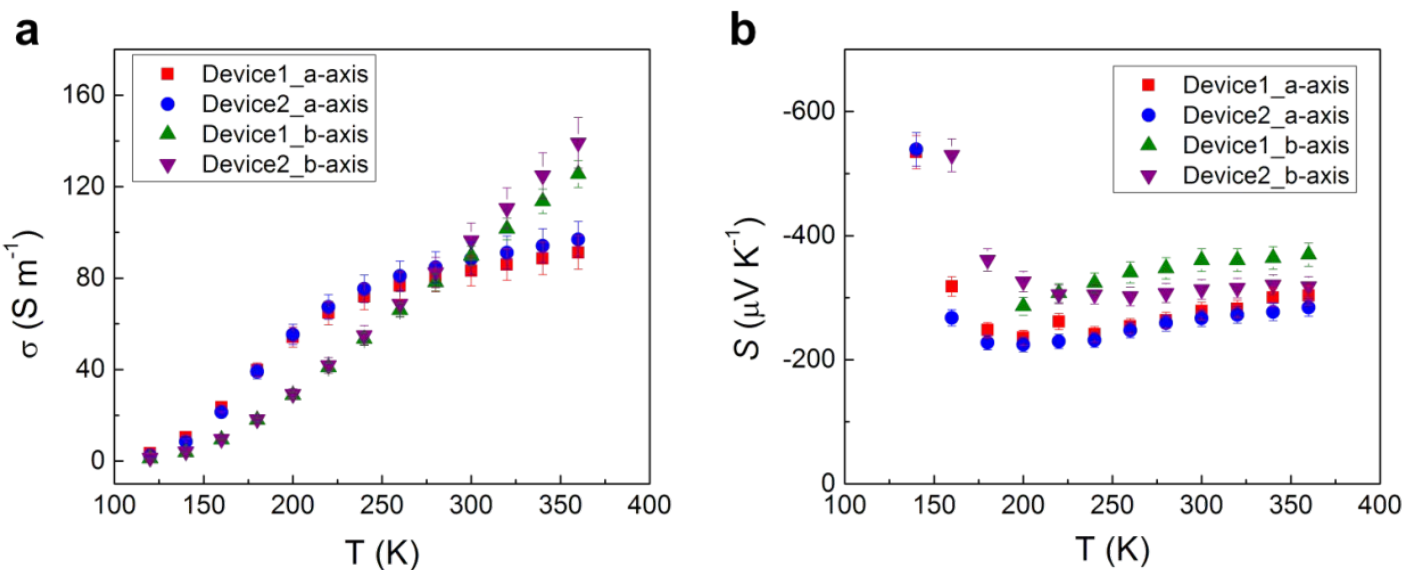

Figure S3. Temperature dependence of electrical conductivity $(\sigma)$ and Seebeck coefficient $(S)$ of $\mathrm{TiS}_{3}$ along its a- and b-axis, respectively. Electrical conductivity shows significant anisotropy between the a- and b-axis. Seebeck coefficient shows a slight anisotropy, behaving as a typical non-degenerate semiconductor.

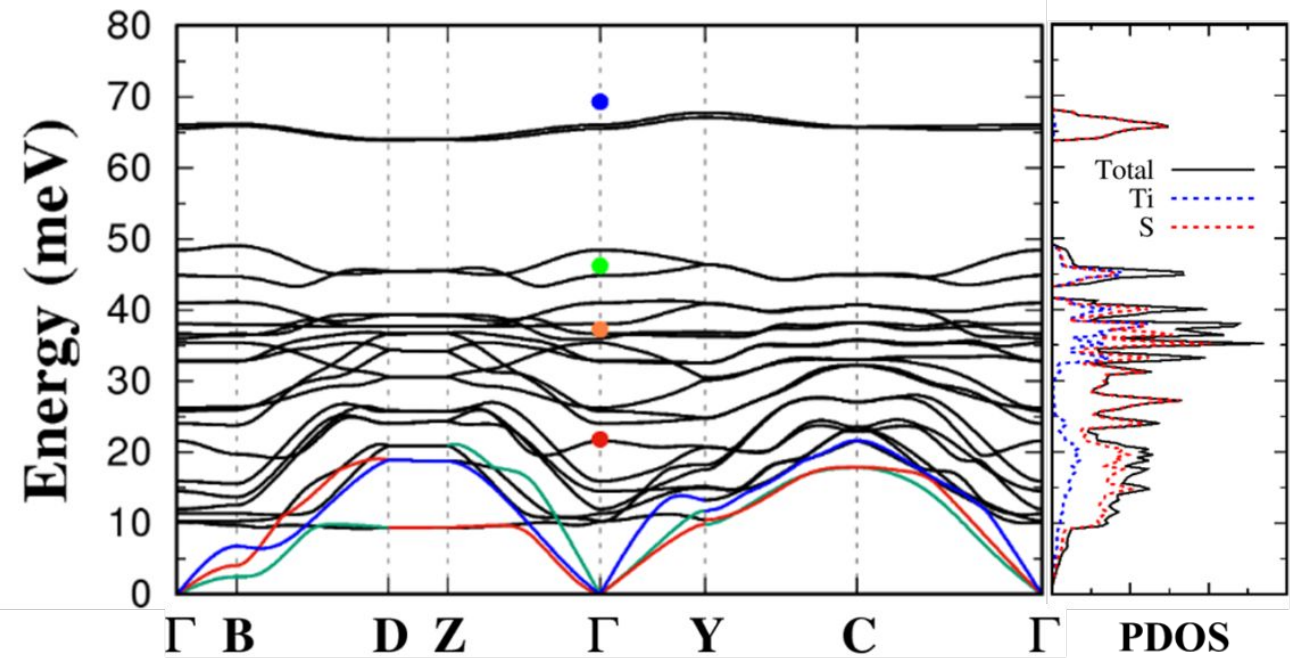

Figure S4. Phonon dispersion of $\mathrm{TiS}_{3}$ calculated along its high-symmetry directions in the Brillouin zone and projected density of states (DOS) of Ti and S. The colored data points are 
experimentally measured Raman peak energies.

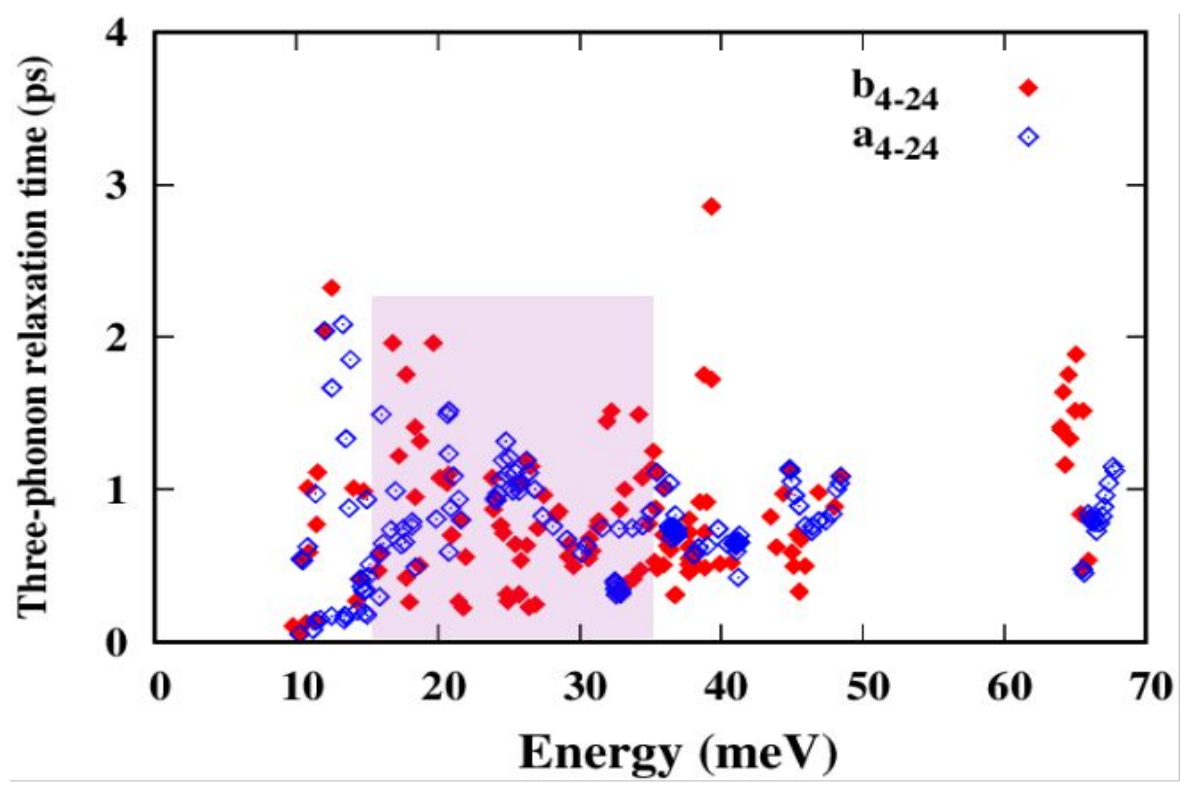

Figure S5. Relaxation time of all three-phonon processes as a function of optical phonon energy for $\mathrm{TiS}_{3}$ at $300 \mathrm{~K} . \mathrm{a}_{4-24}$ and $\mathrm{b}_{4-24}$ indicates contribution from optical phonon branches along the aand b-axis, respectively.

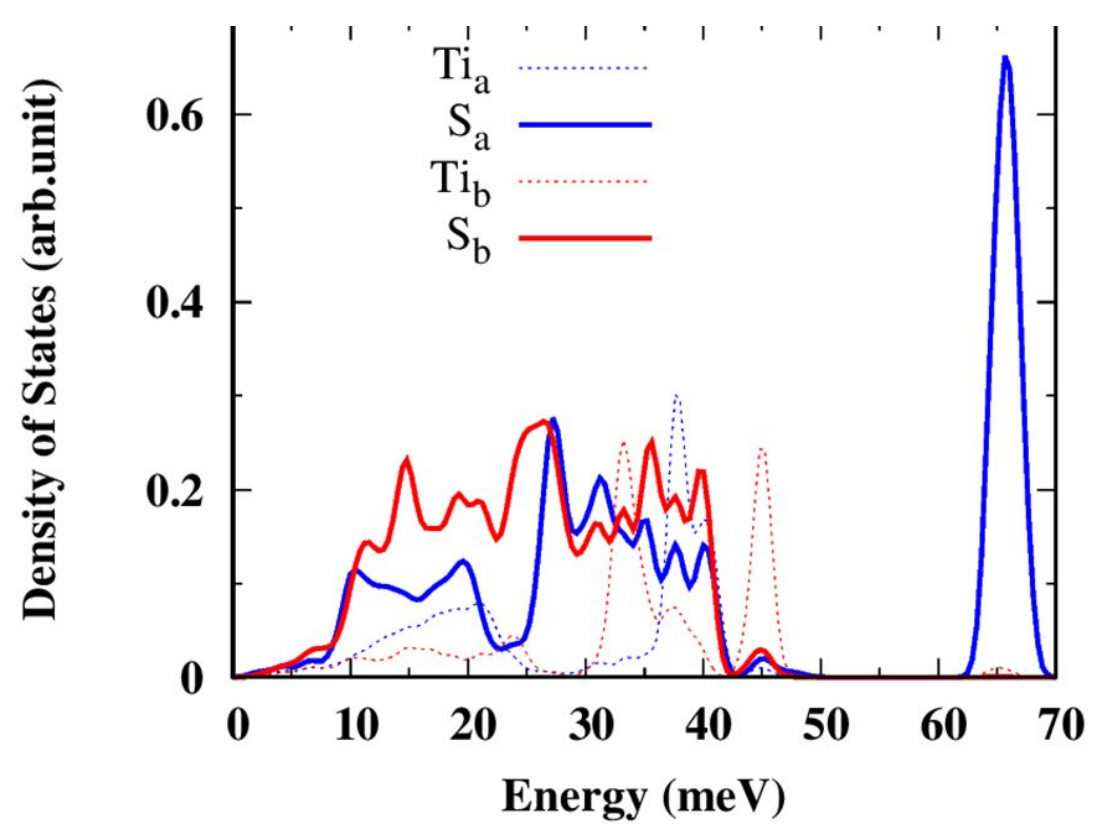


Figure S6. Partial phonon density of states (DOS) contributions from Ti and S atoms for phonons modes propagating along the a- and b-axis, respectively. The anisotropy of DOS from optical phonon modes between $15-30 \mathrm{meV}$ proves that vibrations of $\mathrm{S}$ atoms for phonons propagating along b-axis contribute more to the DOS than that along a-axis.

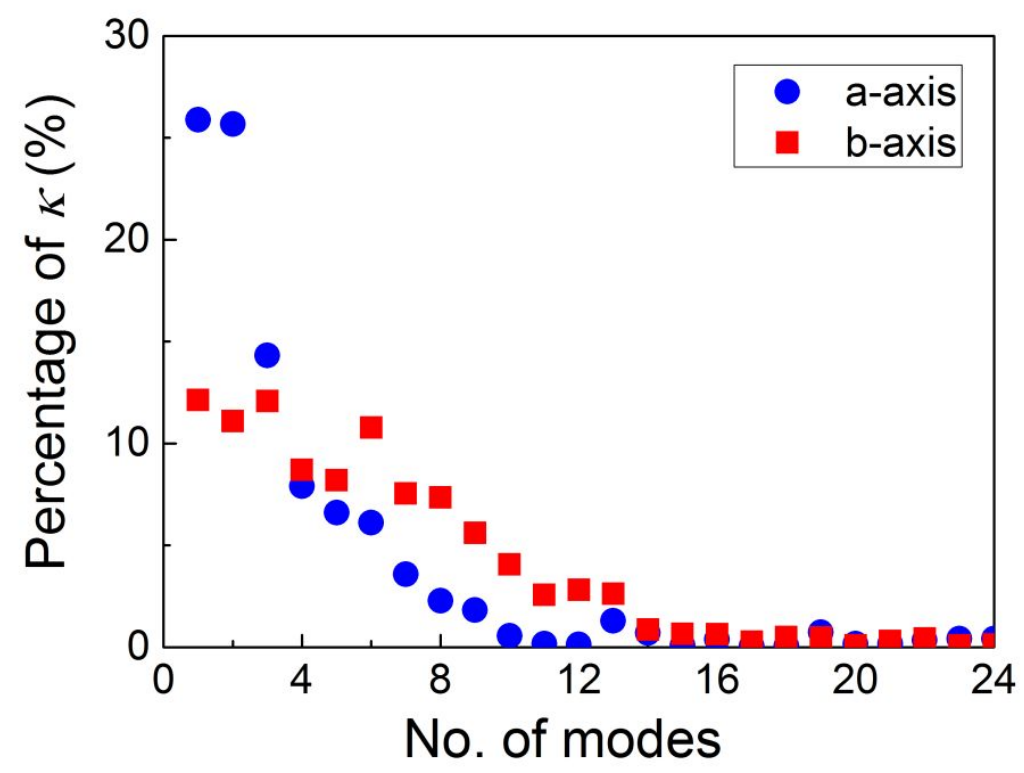

Figure S7. Percentage of contribution to $\kappa$ from different phonon modes along its a- and b-axis, respectively. The optical phonon modes, No. 4-13 ( 15-30 meV), show a significant anisotropy in contribution, where the modes along b-axis contribute more than that of a-axis. 


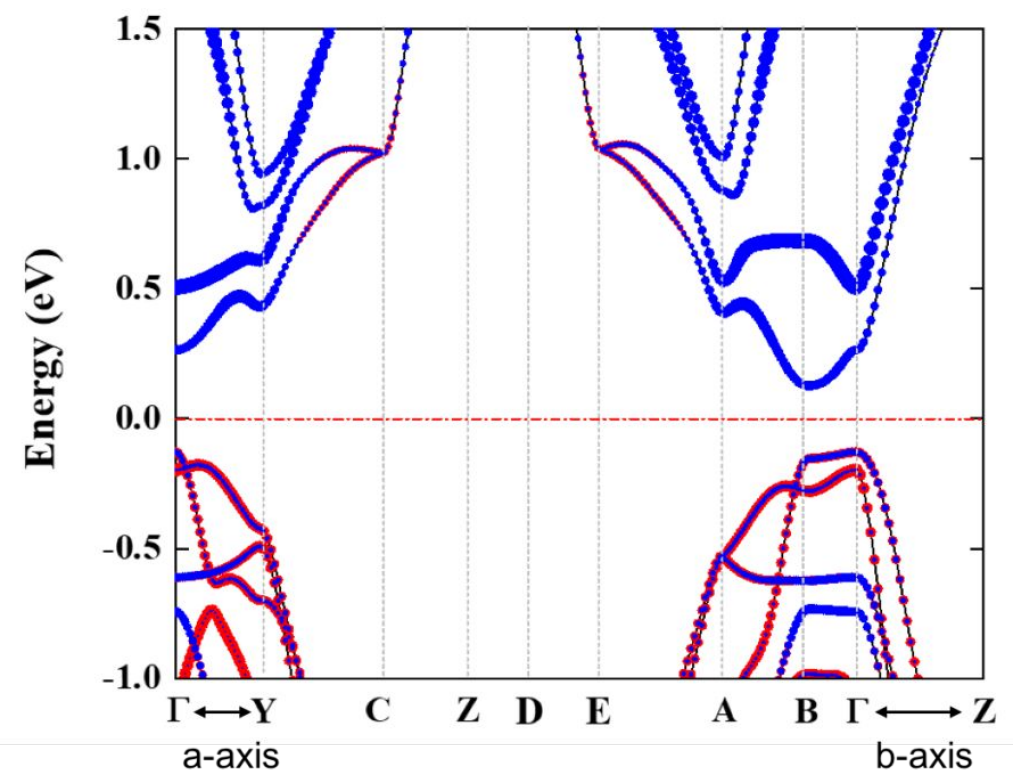

Figure S8. Electronic band structure of $\mathrm{TiS}_{3}$. Blue and red dots indicate the contribution of Ti $3 d$ and S $2 p$ orbitals, respectively. The Ti $3 d$ orbitals dominate the conduction band, dispersing mostly along the b-axis direction.

a

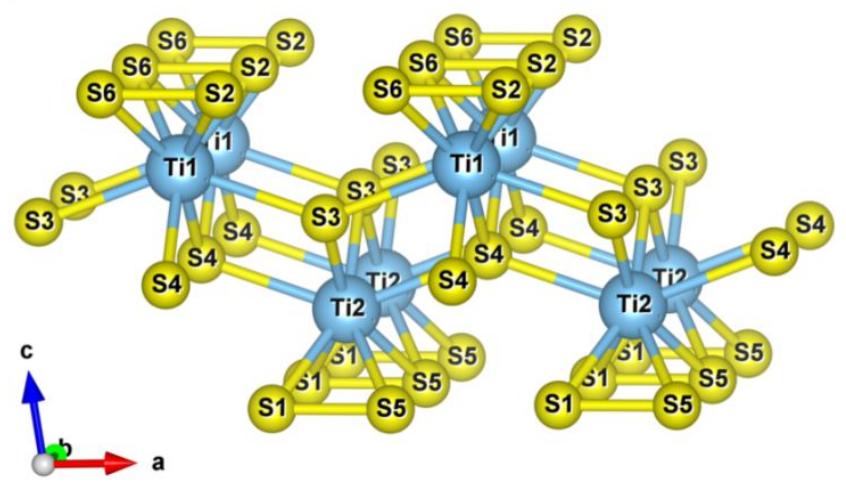

b

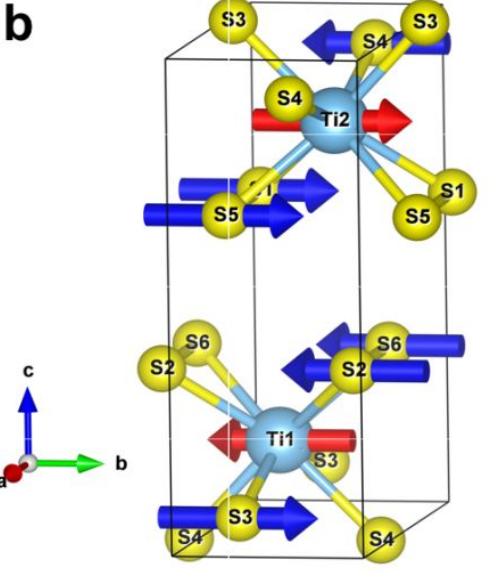

Figure S9. A crystal structure of $\mathrm{TiS}_{3}$. (a) Ti1, S1, S3, and $\mathrm{S} 5$ atoms are equivalent to Ti2, S2, S4, and S6, respectively. (b) The arrows indicate the atomic displacement for Mode 8, the most dispersive optical vibration along the b-axis. 


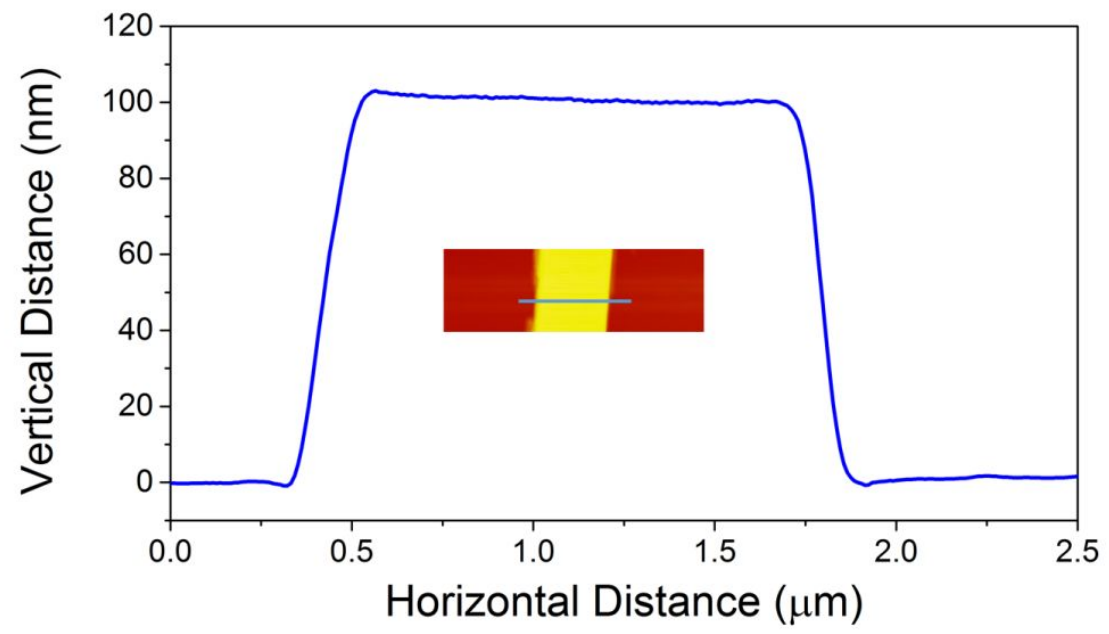

Figure S10. Height profile measured by $\mathrm{AFM}$ for a $\mathrm{TiS}_{3}$ nanoribbon. Inset is an image of the nanoribbon and the line along which the height profile is taken.

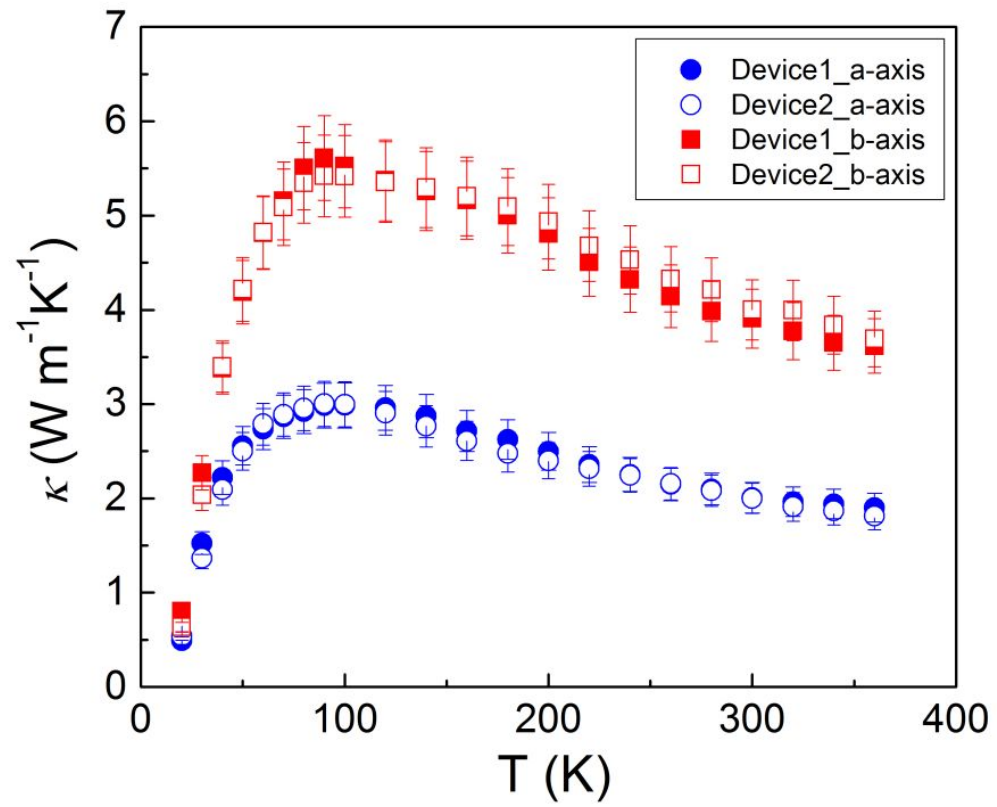

Figure S11. Thermal conductivity of $\mathrm{TiS}_{3}$ nanoribbons along a- and b-axis plotted in linear scale. 

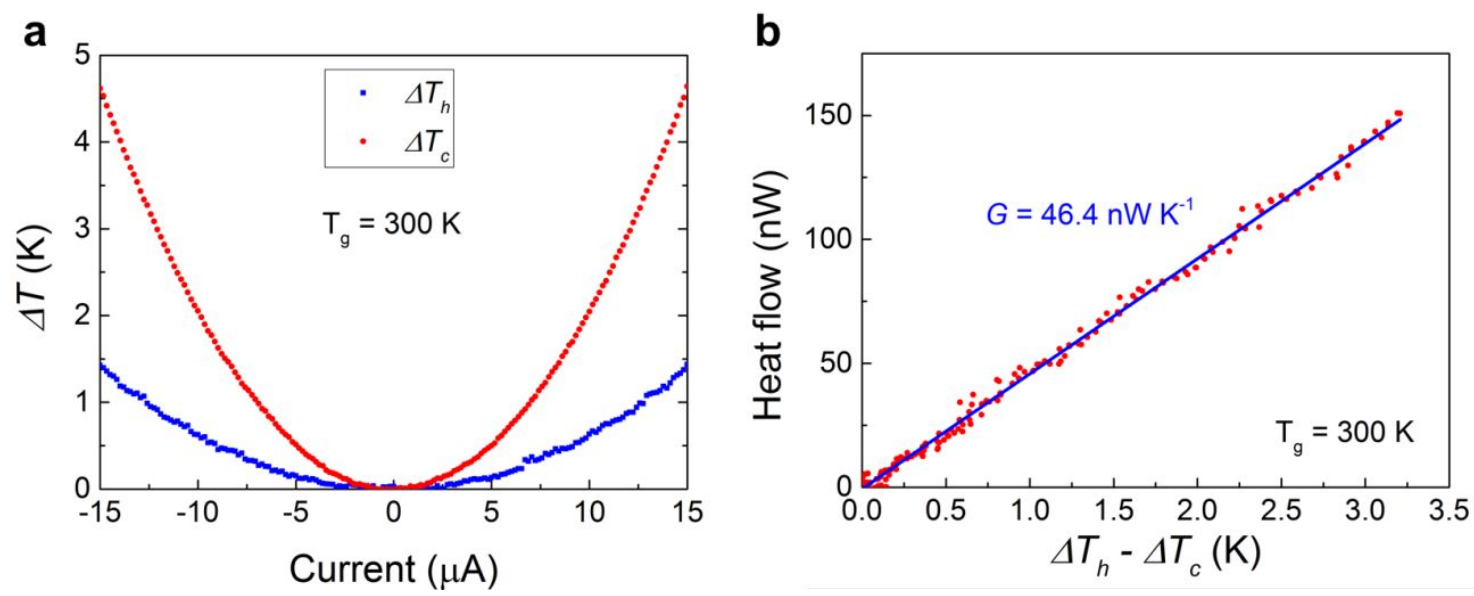

Figure S12. The measured thermal conductance at $300 \mathrm{~K}$ for Device2_b-axis. (a) A raising temperature of hot pad and cold pad as a function of the DC heating current. (b) Heat flow of nanoribbon as function of temperature difference between hot pad and cold pad.

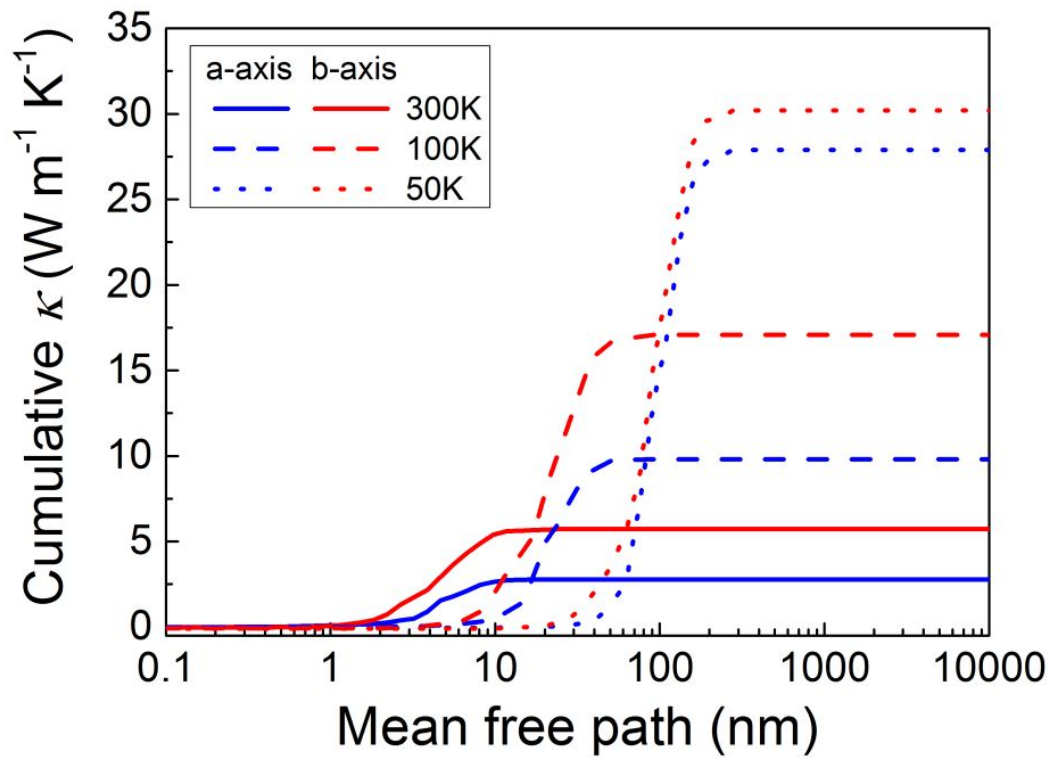

Figure S13. Cumulative thermal conductivity versus phonon mean free path along a- and b-axis at $300 \mathrm{~K}, 100 \mathrm{~K}$, and $50 \mathrm{~K}$, respectively. 


\begin{tabular}{ccccccccccc}
\hline & \multicolumn{4}{c}{ a-axis } & \multicolumn{5}{c}{ b-axis } \\
\hline & $v_{\text {LA }}$ & $v_{\text {TA }}$ & $v_{\text {ZA }}$ & $v_{\text {ave_O }}$ & $v_{\text {LA }}$ & $v_{\text {TA }}$ & $v_{\text {ZA }}$ & $v_{\text {ave_O }}$ \\
\hline \multirow{2}{*}{$v\left(\mathrm{~km} \mathrm{~s}^{-1}\right)$} & Monolayer & 5.43 & 3.01 & 0.88 & - & 6.16 & 2.31 & 1.11 & - \\
& This work & 5.71 & 3.00 & 2.73 & 0.49 & 5.94 & 3.45 & 2.97 & 1.02 \\
\hline
\end{tabular}

Table S1. Group velocity of acoustic phonons (transverse (TA), longitudinal (LA) and out-ofplane (ZA)) and average velocity for the 21 optical phonons $\left(v_{\text {ave_o }}\right)$ near the $\Gamma$ point, predicated by theoretical calculation (this work), compared to that of monolayer $\mathrm{TiS}_{3}$ calculated by Zhang et al. ${ }^{10}$ Group velocities (absolute value average for different $q$ points) are averaged for $q / q_{\max }<$ 0.2 .

\begin{tabular}{|c|c|c|c|c|c|c|c|}
\hline & & & a-axis & & & b-axis & \\
\hline \multirow{4}{*}{$\begin{array}{c}\kappa, 300 \mathrm{~K} \\
\left(\mathrm{~W} \mathrm{~m} \mathrm{~m}^{-1} \mathrm{~K}^{-1}\right)\end{array}$} & $\begin{array}{l}\text { Monolayer, } \\
\text { theoretical }\end{array}$ & & 10.2 & & & 17.9 & \\
\hline & \multirow{2}{*}{$\begin{array}{l}\text { This work, } \\
\text { theoretical }\end{array}$} & acoustic & optical & total & acoustic & optical & total \\
\hline & & 1.87 & 0.97 & 2.84 & 2.04 & 3.74 & 5.78 \\
\hline & $\begin{array}{l}\text { This work, } \\
\text { experimental }\end{array}$ & & 2.0 & & & 4.0 & \\
\hline
\end{tabular}

Table S2. Theoretical and experimental lattice thermal conductivity at $300 \mathrm{~K}$ (this work), compared to that of monolayer $\mathrm{TiS}_{3}$ calculated by Zhang et al. ${ }^{10}$ 


\begin{tabular}{cccccc}
\hline \multicolumn{2}{c}{ Atoms } & Ti1/Ti2 & S1/S2 & S3/S4 & S5/S6 \\
\hline $\begin{array}{c}\text { Born } \\
\text { effective } \\
\text { charge }(e)\end{array}$ & $\left(e^{*}\right)_{\mathrm{aa}}$ & 1.92 & -0.33 & -1.51 & -0.07 \\
\cline { 2 - 6 } & $\left(e^{*}\right)_{\mathrm{bb}}$ & 6.49 & -1.57 & -3.32 & -1.69 \\
\hline
\end{tabular}

Table S3. Calculated in-plane Born effective charge tensor for $\mathrm{Ti}$ and $\mathrm{S}$ in $\mathrm{TiS}_{3}$, where the atoms are labeled in Figure S9a. 